\title{
A mechanistic study of silver nanostructures incorporated reduced graphene oxide via flow synthesis approach
}

\begin{abstract}
Antimicrobials are useful compounds intended to eradicate or stop the growth of harmful microorganisms. The sustained increase in the rates of antimicrobial resistance (AMR) worldwide is worrying and poses a major public health threat. The development of new antimicrobial agents is one of the critical approaches to overcome AMR. However, in the race towards developing alternative approaches to combat AMR, it appears that the scientific community is falling behind when pitched against the evolutionary capacity of multi-drug resistant (MDR) bacteria. Although the "pioneering strategy" of discovering completely new drugs is a rational approach, the time and effort taken are considerable, the process of drug development could instead be expedited if efforts were concentrated on enhancing the efficacy of existing antimicrobials through: combination therapies; bacteriophage therapy; antimicrobial adjuvants therapy or the application of nanotechnology. This review will briefly detail the causes and mechanisms of AMR as background, and then provide insights into a novel, future emerging or evolving strategies that are currently being evaluated and which may be developed in the future to tackle the progression of AMR.
\end{abstract}

Keyword: Antimicrobial resistance (AMR); Current approach; Combination therapy; Nanotechnology; Drug development and microorganisms 